\title{
The Statement of Conjunctive and Disjunctive Queries in Object Oriented Database with Using Fuzzy Logic
}

\author{
Roghayeh Madani \\ Department of Computer Engineering, Ardabil \\ Science and Research Branch, \\ Islamic Azad University, \\ Ardabil, Iran \\ Department of Computer Engineering, \\ Ardabil Branch, Islamic Azad University, \\ Ardabil, Iran
}

\author{
Ali Harounabadi \\ Department of Computer Engineering, Central \\ Branch, Islamic Azad University, \\ Tehran, Iran
}

\begin{abstract}
Entrance of object orienting concept in database caused the relation database gradually to replace with object oriented database in various fields. On the other hand for solving the problem of real world uncertain data, several methods were presented. One of these methods for modeling database is an approach wich couples object-oriented database modeling with fuzzy logic. Many queries that users to pose are expressed on the basis of linguistic variables. Because of classical databases are not able to support these variables, leads to fuzzy approaches are considered. We investigate databases queries in this study both simple and complex ways. In the complex way, we use conjunctive and disjunctive queries. In the following, we use the XML labels to express inqueries into fuzzy. We can also communicate with other sections of software by entering into XML world as the most reliable opportunity. Also we want to correct conjunctive and disjunctive queries related to fuzzy object oriented database using the concept of dependency measure and weight, and weight be assigned to different phrases of a query based on user emphasis. The other aim of this research is mapping fuzzy queries to fuzzy-XML. It is expected to be simple implement of query, and output of execution of queries be greatly closer to users' needs and fulfill her expect. The results show that the proposed method explains the possible conjunctive and disjunctive queries the database in the form of Fuzzy-XML.
\end{abstract}

Keywords: Object Oriented Database, Fuzzy Database, Fuzzy Object Oriented Database, Fuzzy query, Tag XML.

\section{INTRODUCTION}

Relational database model doesn't satisfy the need of users that intend to work with uncertain data. Therefore, they combined fuzzy approach (to support uncertainty and linguistic variable) and object-oriented approach to each other and created a powerful database in the name of objectoriented database. The fuzzy object-oriented database models in a logical level afford with data uncertainty as well as combined objects existing in the real world. Upon defining some main concepts of database such as objects, classes, object-class relations, subclass-superclass and inheritance under fuzzy environment, a few queries can be propounded to giver more well-suited responses. The responses in this type of database with a degree of membership may belong to the response range and this subject is very useful, because the responses may be limited to a threshold range and return results to the user so that their closeness to the response to be appropriate for that user, whilst in the classic database, no user is able to do it and could observe responses that exactly satisfied all query conditions, and not a part of conditions. This inflexibility for returning the responses always bothered the users.

What is important in the meantime is quality of queries expression for combination of two object-oriented and fuzzy approaches. The queries must be expressed benefitting from language variables and the objects appearing in the response are graded (sorted) by belonging degree.

In second part of this paper, the background and in third part related works and in fourth part, proposed method were presented. In the proposed method of this paper, fuzzy queries in addition to being expressed by disjunctive and conjunctive may be stored by XML labels. Fifth part was allocated to case study and sixth part to conclusion.

\section{BACKGROUND}

Background of this study includes fuzzy set, fuzzy objectoriented database, XML.

\subsection{Fuzzy sets}

Fuzzy sets and fuzzy logics theory was introduced in 1965 by Professor zadeh. As a result, in 1977, possibility theory was founded. This theory is used for encountering most real world phenomena therein uncertainty exists [1].

In the classic logic, membership in a set is assumed as zero and one so that if a member exists in a set is shown by 1 and otherwise by 0 . In fact, membership degree is a function that its range is member of $\{0,1\}$. On the other side, in fuzzy logics, the concept of membership degree in a set is extended to range $[0,1]$. The concept of fuzzy logics is taken into consideration because in the real world, plenty of man arguments and reasons are uncertain and approximate.

\subsection{Fuzzy object oriented database}

In order to simulate uncertain data and multivalued attributes as well as complex relationships between objects in the object oriented database, current efforts are focused on conceptual data model and object oriented database with uncertain data. 
For the first time, Milano and Zicari (1990) introduced incomplete information about uncertain data modeling in object oriented database, in the name of null [2].

From then on, combination of uncertain data in object oriented database was noticed increasingly, there in ambiguity in sample objects and class hierarchy is observable. Encapsulating inaccurate and unspecified information in the database model was one of important subjects in the database researches. Therefore, fuzzy object oriented database (FOOD) is presented to afford with inaccurate and complex objects in the real world in a logical level. Some main concepts such as objects, classes, objects-classes relationships, subclass, superclass and polymorphisms are extended in FOOD under fuzzy data environment. Ultimately, an overall model is presented for FOOD.

\subsection{XML (extensible markup language)}

This language was established in 1996 by web consortium (W3C) to eliminate the existing restrictions in HTML and adding new options [3]. XML is a simple subset of standard generalized markup language (SGML) that is a general and complex for marking the data. XML is able to keep the structure of stored data along with their meaning. Features of $\mathrm{XML}$ and its elements converted this language to portable and standard language for different users. From one side, XML upon storing its files in textual format and using markup signs makes the stored data understandable for man and on the other hand, provides these files in structured form to the programs to simplify its processing for computer [4].

\section{RELATED WORKS}

Recently, a lot of works have been created for fuzzy data modeling and working on object oriented database. Medina et al [5] to implement the extended fuzzy relational database show the needed elements. Basis for this database is a relational database that was extended for including fuzzy data. The most important factor of this process is simplicity of implementation.

Ma Zoung could develop an object oriented database model based on possibility distribution and semantic criterion of fuzzy data to use inaccurate and complex objects in the real world. In addition, a few main concepts in object oriented database such as objects, classes, object-class relationships, subclass-superclass and polymorphism were extended under fuzzy data environment [6].

Harounabadi et al [7] used uncertain systems for modeling. This extended version includes the both structure and behavior aspects in the system.

In [4], fuzzy extension of XML was proposed for modeling inaccurate data.

Cavalcante et al [8] offered new fuzzy database architecture. Commonly, fuzzy database stores the data and metadata to incorporate a concept in the name of total range of different storable samples in this database. They introduced a new method for offering fuzzy metadata base that simplifies the tasks of data management. The main advantage of this new exhibition is simplicity in understanding, implementation, use and support. This new metadata base organizes the data using XML format that adds more advantage to probability.

Gupta et al [9] presented a method for use of fuzzy data in a traditional database.

Pourbehzadi [10] allocating a weight to different parts of query and possibility of determining accepted threshold value for each queried attribute, allows the user to extract its needed objects from database and presents a model that explains fuzzy query as more significant responses and closer to the query.

\section{THE PROPOSED METHOD}

The proposed method is that fuzzy queries are mapped to fuzzy-XML. An object oriented database is designed and fuzzy values are applied thereon and on the queries. In this paper, fuzzy queries in addition to being expressed by disjunctive and conjunctive proposition have the possibility of being stored by XML labels. Upon combining XML labels and fuzzy-making techniques, labels may be presented for support of fuzzy object oriented database queries.

\subsection{Definition of class structure in XML document}

To define class structure in XML, respective class must be shown in XML schema. For this purpose, class exhibition must be mapped to DTD schema.

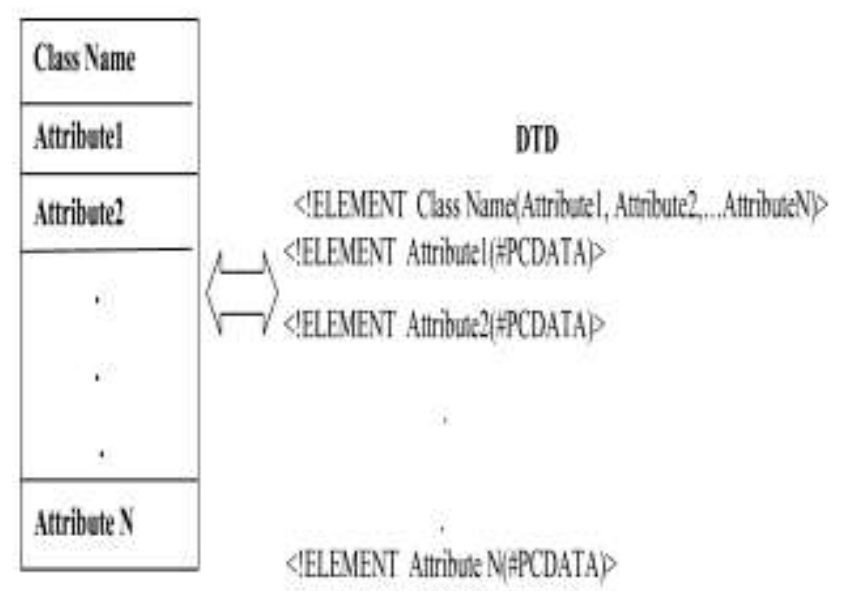

Figure 1. Class mapping by DTD

\subsection{Exhibition of samples of class in XML document}

Whereas class was formed based on XML schema, at this stage, the defined samples of class may be easily shown in XML document as below:

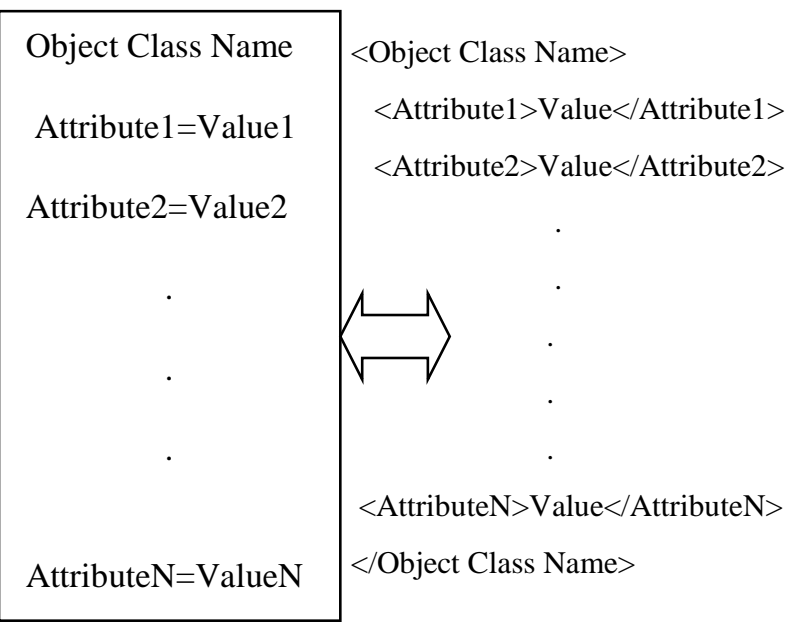




\subsection{Exhibition of fuzzy data in XML documents}

XML data is shown in a special structure and aiding XML ambiguous and uncertain data may be exhibited using intrinsic characteristics of XML. Fuzzy values have the capability of showing uncertain data and are exhibited aiding a defined fuzzy structure in XML.

To show the concept of uncertainty in XML, solutions must be presented for attributing membership degree to element as well as possibility distribution of elements attributes. At first, the concept of attributing a membership degree to XML element must be specified. This subject may have ambiguities because elements may be placed in nested and hierarchical form, and each one of elements has membership degree. In fact, membership degree of each element shows that element's occurrence possibility and all subtree elements with the root of the same element. Figure 2, shows a membership function that has two linguistic variables:

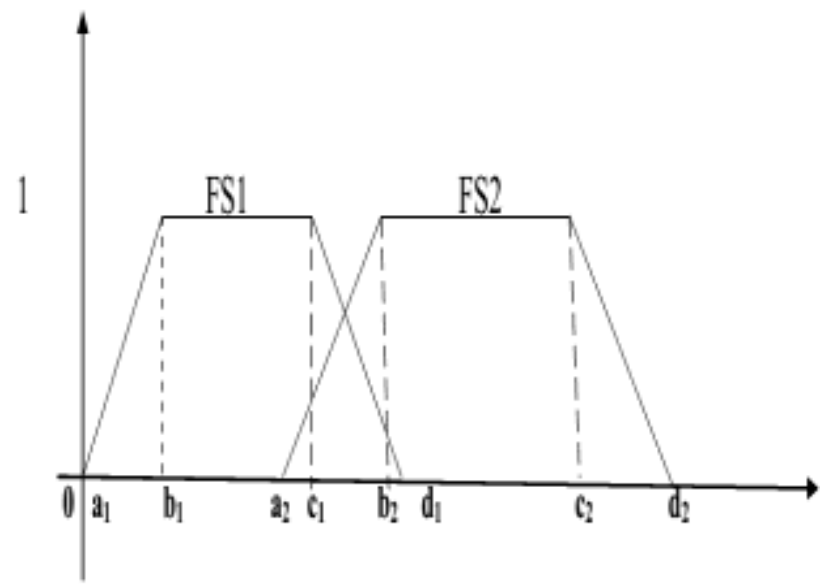

Figure 2. membership function diagram

In consideration of membership function as per figure 2, an exhibition of that function may be in XML document.

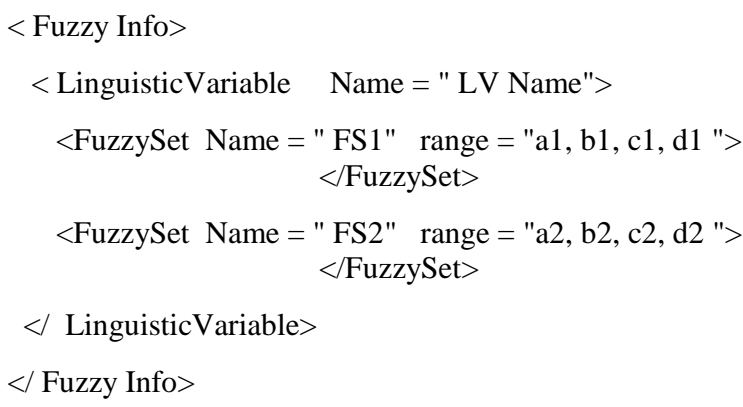

$</$ LinguisticVariable >

$</$ Fuzzy Info>

\subsection{Query procedure}

Query processing in fuzzy object oriented database refers to a procedure therein objects are selected from classes that simultaneously satisfy the given threshold as well as satisfy given condition in the condition threshold. It is obvious that queries have threshold in fuzzy object oriented database that are connected to choices of threshold.

In the method proposed by Ma Zoung, it was not possible for the user to prioritize its respective attributes in query and only membership of each object in relative class $(\mu)$ as well as fuzzy degree of respective attribute was analyzed.

In the proposed method of this paper, upon allocating the weight to queried attributes, determination of priority of each attribute in query is assigned to the user. Therefore, syntax rule of a SQL query based on fuzzy object oriented database model is as follows:

SELECT < attribute list> FROM <class $1, \ldots$, class $_{\mathrm{m}}>$ WHERE <query condition WITH threshold>

In the above equation, attribute list includes attributes that are going to be appeared in the output and related to the objects that satisfy he user condition. The classes that are written in front of From include those that browsing operation is implemented there in. Query condition is a fuzzy condition that user expects to be satisfied in the respective threshold mentioned in front of it. Furthermore, all thresholds include numbers within range $[1,0]$. Queries related to fuzzy object oriented database may be written in XML format that a sample thereof is shown in figure 3 :

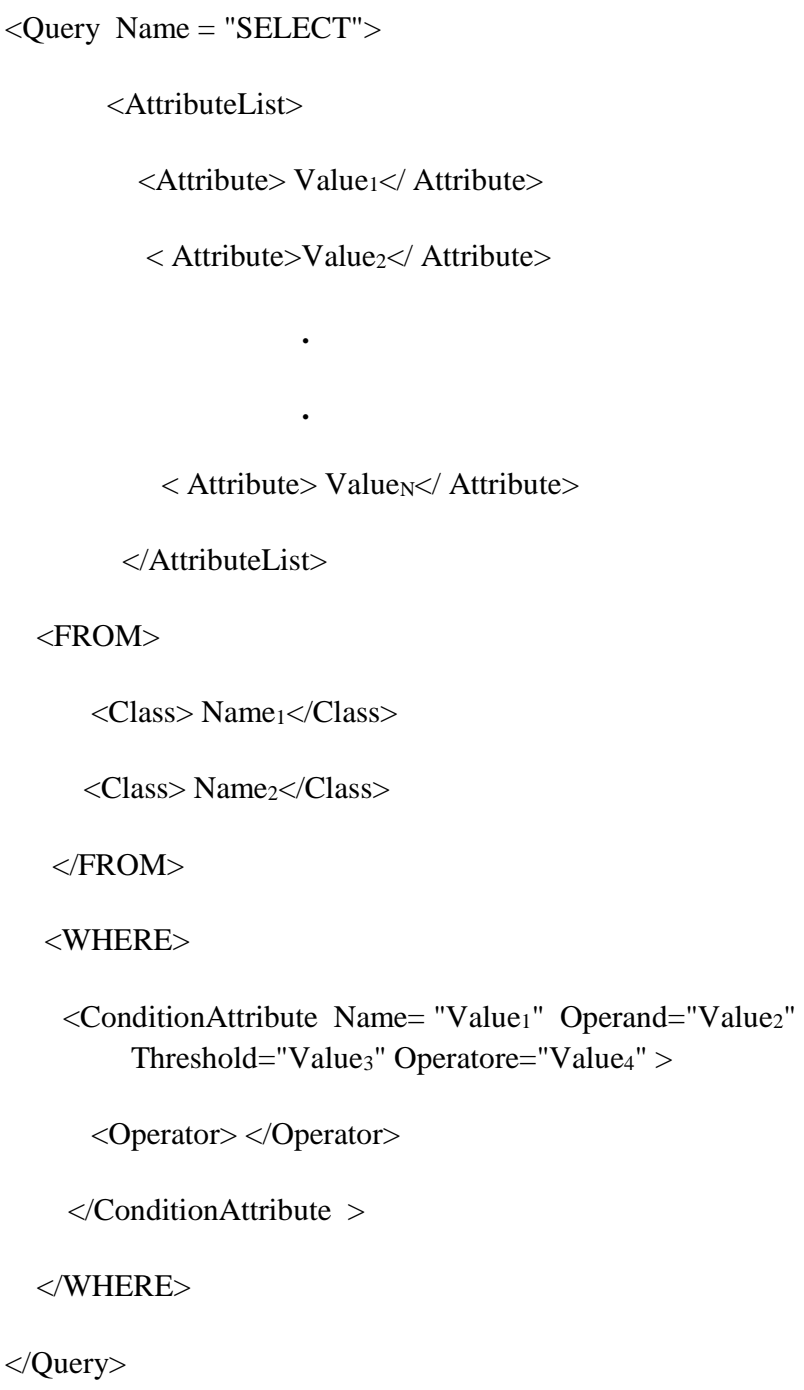

Figure 3. Exhibition of Select query using XML 
The user should define three cases for each query condition:

1. The amount of an attribute (which can be fuzzy such as age attribute and amount "teenager")

2. Threshold of attribute membership degree (for example, with a threshold of 0.6 it can belong to teenager class)

3. Attribute weight (this weight represent the level of importance or priority of each attribute for the user).

As soon as the query by user, final threshold value is calculated using values appeared in query and following equation:

$$
\text { Threshold }_{\text {final }}=\frac{\sum_{i=1}^{n}\left(\mathrm{~T}_{i} * W_{i}\right)}{\sum_{i=1}^{n}\left(W_{i}\right)}
$$

In above equation, $T_{i}$ denotes defined threshold for attribute $\mathrm{i}$ and $\mathrm{W}_{\mathrm{i}}$ denotes the respective weight for attribute $\mathrm{i}$ in the query.

Now, the requested $\mu$ values are calculated for each object separately and using functions written formerly in the database and in the class of said object. After calculating it is multiplied by $\mathrm{W}$ related to same attribute raised in the query and so $\mu_{\text {final }}$ value is calculated for each object using following equation:

$$
\mu_{f i n a l}(\text { Obja })=\frac{\sum_{i=1}^{n}\left(\mu_{i} * W_{i}\right)}{\sum_{i=1}^{n}\left(W_{i}\right)}
$$

In the above equation, $\mu \mathrm{i}$ is the degree of belonging object a to an attribute $1, \mu \mathrm{j}$ is the degree of belonging object a to attribute 2 and $\mu \mathrm{k}$ is the degree of belonging object a to attribute $\mathrm{n}$ and $\mu$ final (obj.a) is the final belonging degree of object a to the set of answers. In this stage, for any object we have a degree of belonging and we should compare this degree of final belonging to the query required threshold and if the degree of final belonging is higher than the query threshold or equal to it, we can bring this object in the answer set. It means only the objects will be appeared in the answer set where they achieve the mentioned condition in following equation:

$$
\mu_{\text {final }} \geq \text { Thresholdfinal }
$$

\section{Case study}

In the case study of this paper, book information registration system has been taken into consideration. In this system, the user registers the books through entering initial information of book together with its author's name in the database of library. In this system, possibility of fuzzy search based on weight and price or the both is provided and the user will have the fuzzy search possibility based on weight, price or the both.
The classes related to book information registration system include two classes Book and Author that its specifications are as follows:

Class Book \{

Int ID;

String Title;

String ISBN;

String Publisher;

Date Date;

double Price; // Fuzzy Linguistic Variable

Float Weight; // Fuzzy Linguistic Variable

String Subject;

Author Author; //Object as Data in Object Oriented Database \}

Class Author \{

String FirstName;

String LastName;

\}

According to the defined classes and proposed method, a mapping to DTD model must be applied to obtain XML document related to respective system, easily.

$<$ !ELEMENT Book(ID, Title, ISBN, Publisher, date, Price, Weight, Subject, author)>

$<$ !ATTLIST Book ID CDATA \#REQUIRED>

$<$ !ELEMENT Title (\#PCDATA)>

$<$ !ELEMENT ISBN (\#PCDATA)>

$<$ !ELEMENT Publisher (\#PCDATA)>

$<$ !ELEMENT Date (\#PCDATA)>

$<$ !ELEMENT Price (\#PCDATA) >

$<$ !ELEMENT Weight (\#PCDATA)>

$<$ !ELEMENT Subject (\#PCDATA)>

$<$ !ELEMENT Author(FirstName, LastName)>

$<$ !ELEMENT FirstName (\#PCDATA)>

$<$ !ELEMENT LastName (\#PCDATA)>

Considering fuzzy data in the studied system including price and weight features, membership functions are shown as below: 


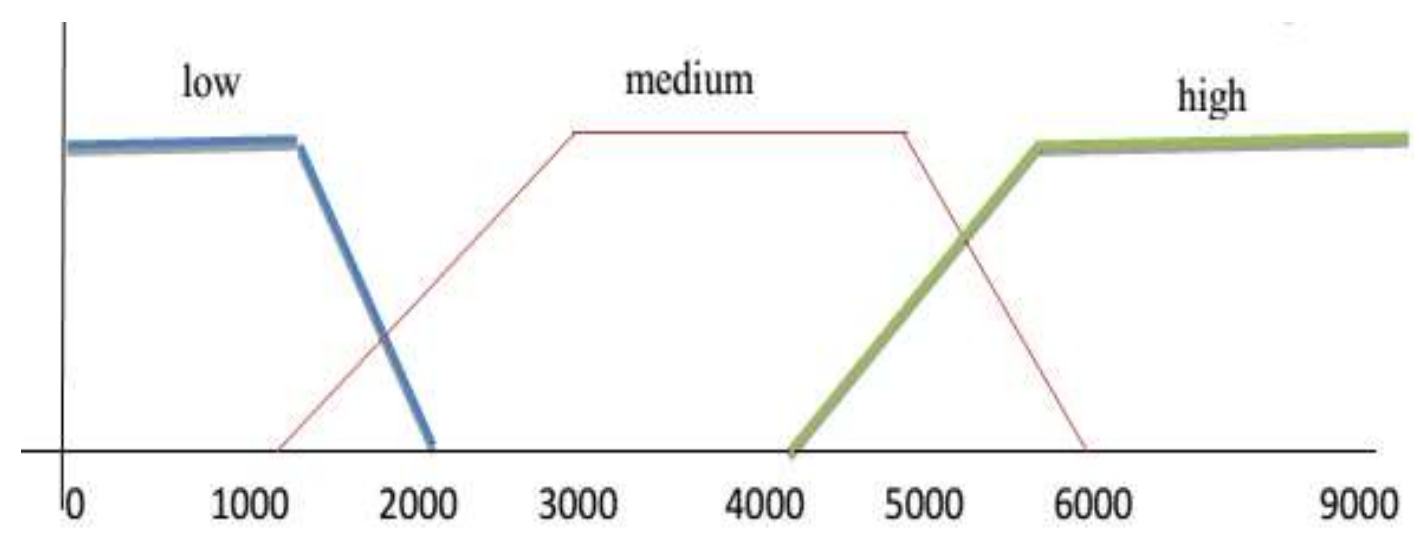

Figure 4. Membership function of price linguistic variable

<LinguisticVariablename="Price">

<FuzzySetname="Low"range="0 01000 3000"> </FuzzySet>

<FuzzySetname="Medium"range="2000 30005000 6000"> </FuzzySet $>$

<FuzzySetname="High"range="4000 60009000 9000"> </FuzzySet> $</$ LinguisticVariable $>$

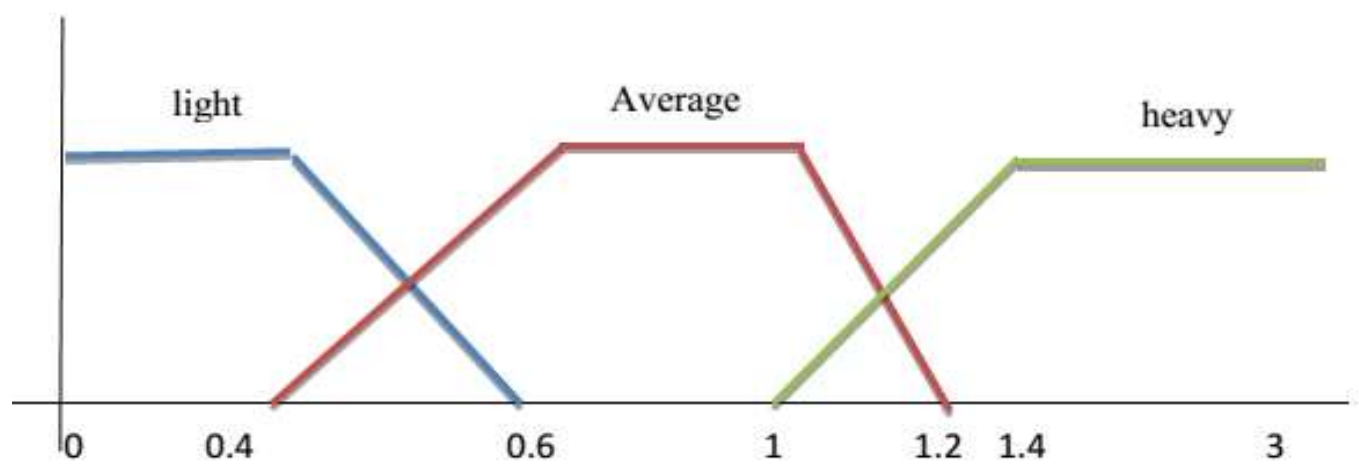

Figure 5. Membership function of weight linguistic variable

<Linguistic Variablename="Weight">

<FuzzySetname="Light"range=" $00.4 \quad 0.6 "></$ FuzzySet $>$

<FuzzySetname="Average"range="0.4 0.611 .2 "></FuzzySet>

<FuzzySetname="Heavy"range="1 1.43 3" 3 ></FuzzySet>

$</$ LinguisticVariable $>$

A sample of fuzzy search environment related to the studied system is exhibited in the following figure. As per this figure, search operation is performed based on field and value (threshold range). 


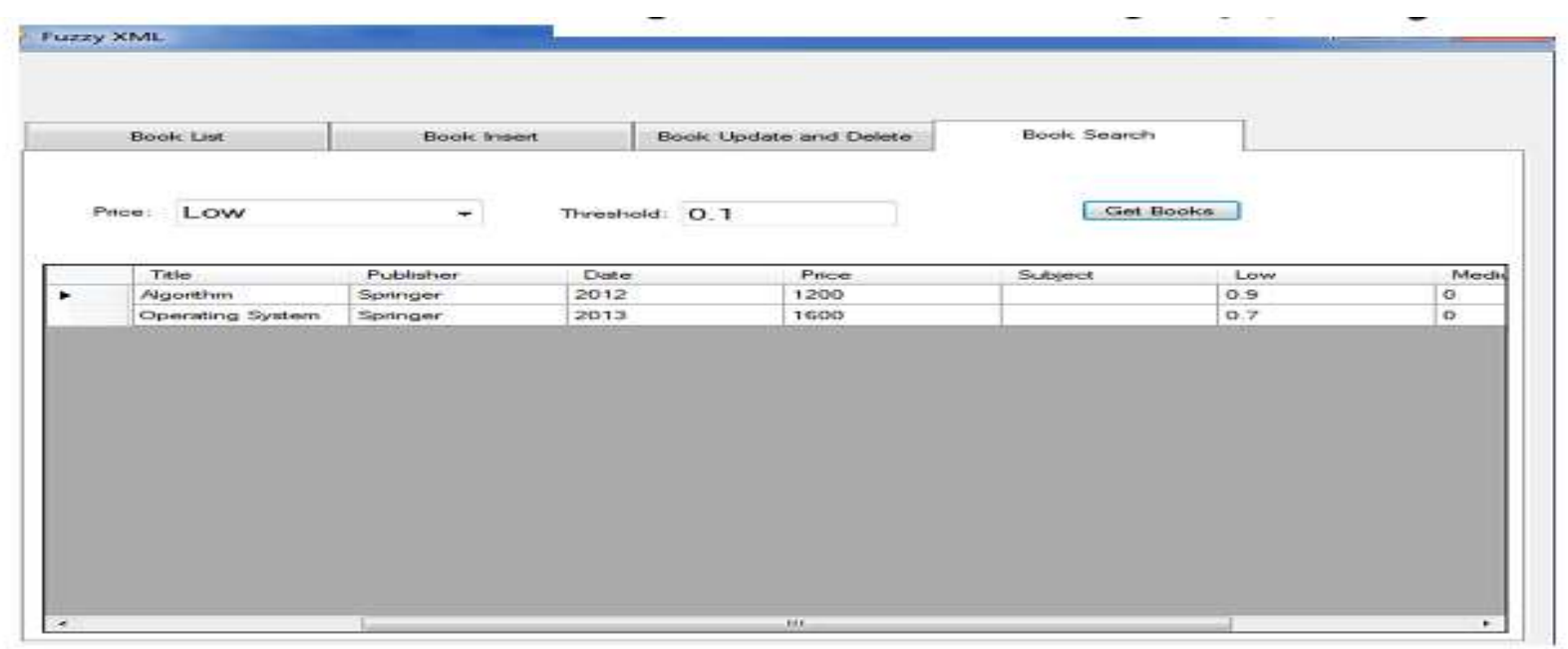

Figure 6. Display form of fuzzy search result

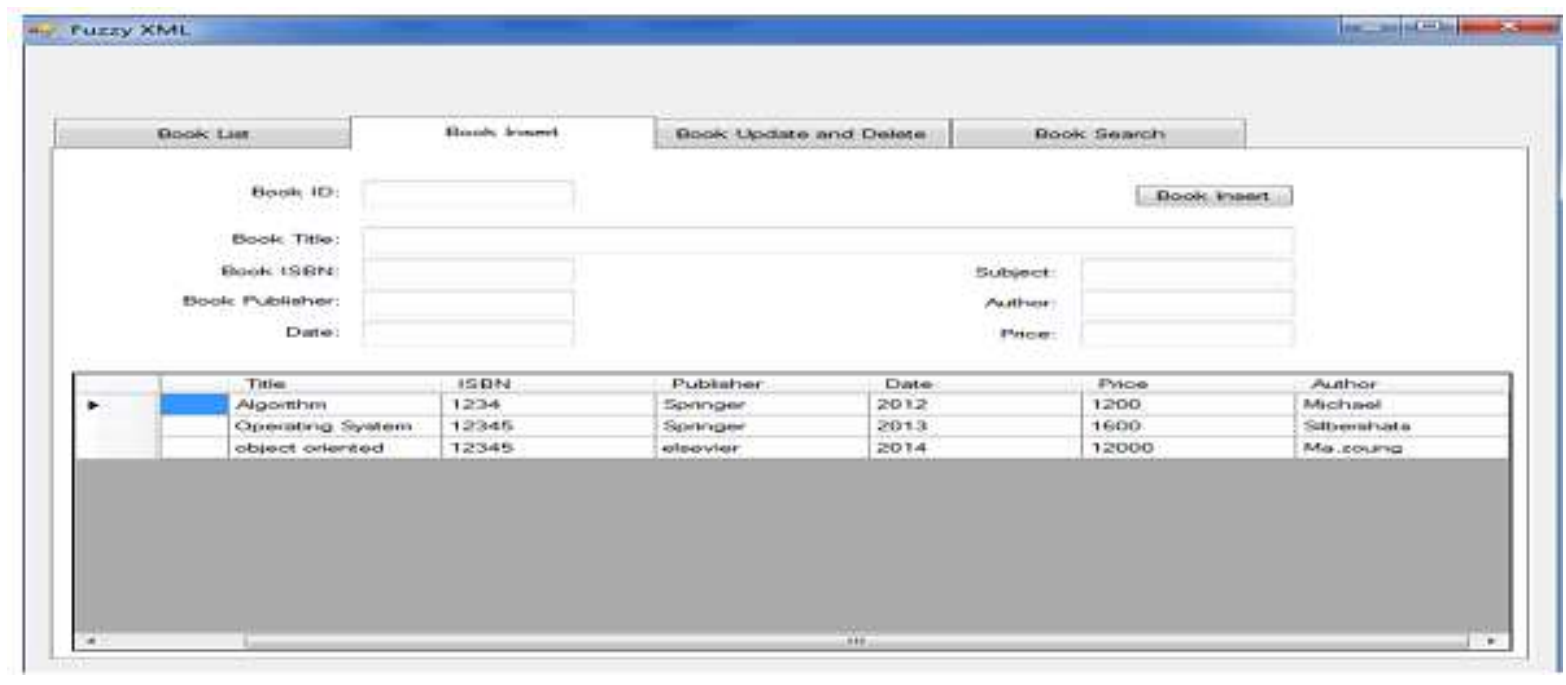

Figure 7. Display form of book insertion

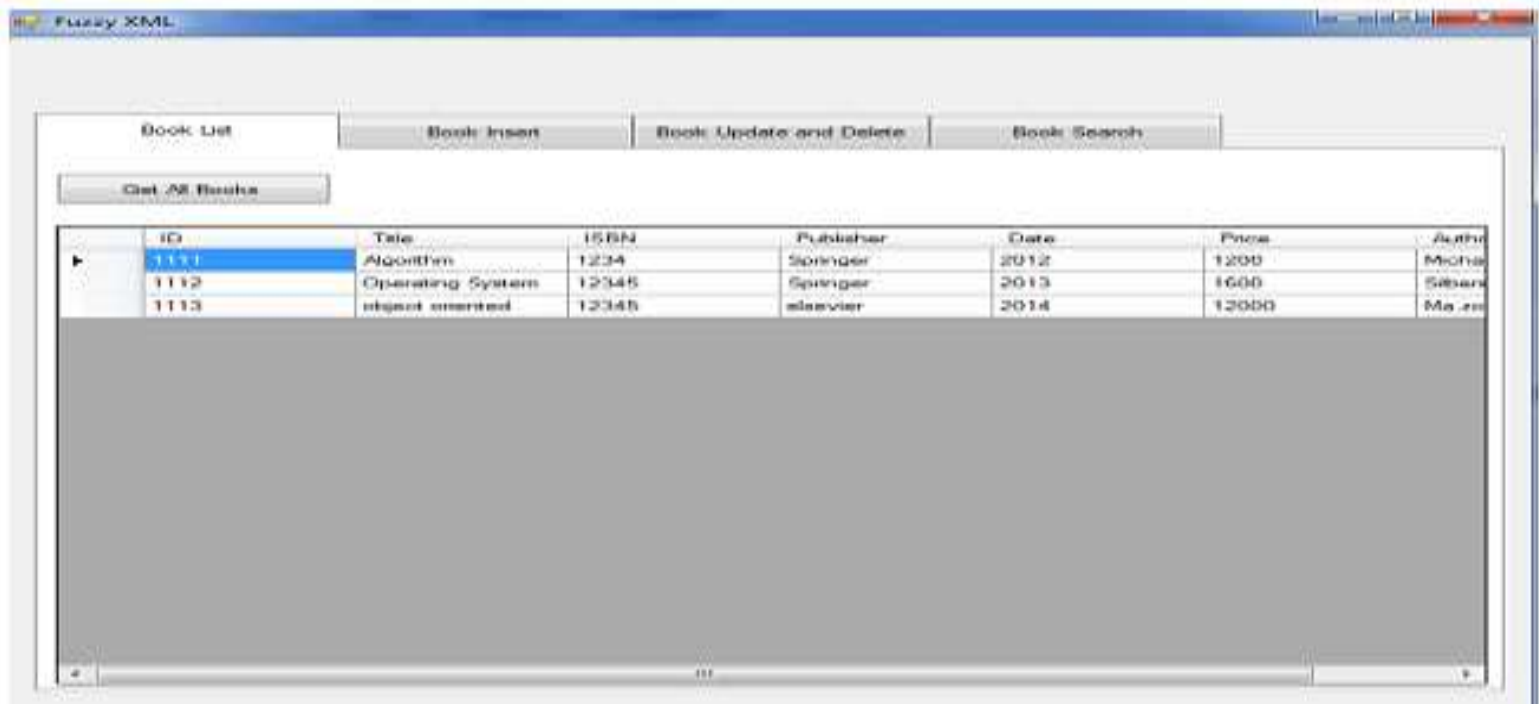

Figure 8. Display form of book list 


\section{Conclusion}

In this paper, a method was implemented for improvement of fuzzy queries in the object oriented database. Fuzzy queries in addition to being expressed by disjunctive and injunctive proposition, can be stored with XML labels. The queries in the object oriented database were amended using the concept of membership degree and weight. Fuzzy data was stored as XML tags and fuzzy queries converted to Fuzzy-XML.

\section{REFERENCES}

[1] Zade, L. A. 1978. Fuzzy sets as a basis for a theory of possibility, Elsevier, Fuzzy sets and Systems, vol. 1, pp. 3-28.

[2] Zicari, R., Milano, P. 1990.Incomplete information in object oriented databases, ACM SIGMOD record, vol. 19, Issue 3, pp. 5-16.

[3] Tim, B., Jean, P., Sperberg, C. M., Eve, M. 2006.ExtensibleMarkup Language (XML)1.0. World Wide Web Consortium (W3C). http://www.w3c.org/TR/REC-xml. Fourth Edition, 29 September. (Visited on 2008-02-09).

[4] Ma , Z. 2005.Fuzzy Database Modelling With XML. Springer publishing.

[5] Medina, J. M., Vila, M. A., Cubero, J. C., Pons, O. 1995. Towards the implementation of a generalized fuzzy relational database model, Fuzzy sets and Systems, vol. 75, Issue 3, 10 November 1995, pp. 273-289.

[6] Ma , Z. 2005.Fuzzy Database Modelling of Imprecise and Uncertain engineering Information.Studies in Fuzziness and soft Computing,195,137-158.

[7] Haroonabadi, A., Teshnelab, M. 2009. Behavior Modeling in Ucertain Information System by Fuzzy-UML. International of soft Computing,4(1), 32-38.

[8] Cavalcante, R.T., Cruz, A.J.O., Rodrigues, R.D., Aliança. 2009. A proposal for a fuzzy database architecture incorporating XML. Elsevier, Fuzzy Sets and Systems, Volume 160, Issue 2, PP. 269-279.

[9] Gupta, p., Rishi, R.,\& Mittal, H. 2011. Database Desighn for Storage of Fuzzy Information in Traditional Databases. International Journal of Computer Applications, 15(2).

[10] Pourbehzadi, M., Hrounabadi, A., Sadegzadeh, M. 2012. A new weighted fuzzy grammer on object orientet database queries, Growing Science. 